\title{
THE FLORAL CHARACTERISTICS OF THE ROAD EDGE EFFECT ON THE EXAMPLE OF A ROAD WITH LOW TRAFFIC VOLUME IN THE "PUSZCZA NOTECKA" FOREST
}

\author{
Paweł Mizera ${ }^{\bowtie}$, Sylwester M. Grajewski, Adrian Kasztelan \\ Department of Forest Engineering, University of Life Sciences in Poznań \\ Mazowiecka 41, 60-623 Poznań, Poland
}

\begin{abstract}
This paper concerns the edge effect of a provincial road on the broadly understood inventory characteristics of the neighbouring inland dune pine stands in the "Puszcza Notecka" Forest in Poland. The observations were conducted in the pine stands in the fresh coniferous forest and fresh mixed coniferous forest sites. Twelve rectangular experimental sites, called belt transects, were established perpendicular to the road axis. Each of them consisted of 3-4 plots located at different distances from the pavement edge. In the course of the survey of the flora, a total of 108 plant taxa, out of which $70 \%$ are found solely in the immediate vicinity of the road, $25 \%$ were found both along the road and in the stand interior and $5 \%$ were found only inside the stand. The shrub layer and the forest floor vegetation layer showed a higher coverage and species richness with the opposite trend observed for mosses and lichens. Despite the significant participation of synanthropic plants, there was no invasion of alien species. Due to the low volume of traffic and the lack of maintenance treatments of road surface, the reasons of changes in the species composition are attributed to the modifications of light and humidity conditions.
\end{abstract}

Key words: forest roads, Scots pine, pine forests, vegetation cover

\section{INTRODUCTION}

The impact of transportation routes on the environment is a very broad problem and it is frequently investigated by experts from various disciplines of life such as engineering and economic sciences (see eg. Bignal et al., 2007; Czerniak et al., 2007; Eigenbrod et al., 2008; Forman and Alexander, 1998; Garcia-Gonzaleza et al., 2012; Kuitunen et al., 2003; Koivula and Vermeulen, 2005; Valentukevičienè, 2016). The results of such research indicate that an ecotone zone is formed at approx. 30-100 (200) m width in the immediate vicinity of a road (Marcantonio et al., 2013). However, the most characteristic changes in the physiognomy are observed in the 15 (20) $\mathrm{m}$ wide belt adjacent to the stand wall. They results from the changes in lighting, moisture content, wind force, soil fertility, noise, vibrations as well as air and soil pollution etc. (Bernhardt et al., 2004; Corney et al., 2006; Delgado et al., 2007; Hawbaker et al., 2006; Suárez-Esteban et al., 2013).

In the opinion of Faliński (1961), the vegetation along forest roads exhibits many characteristics of synanthropic, particularly ruderal, vegetation. The synanthropisation processes are evident especially at the boundaries of forest complexes and at the edges of transportation routes (Balcerkiewicz and Kasprowicz, 1989). Those transition zones are frequently referred 
to as the ecological corridors or margins (Koivula and Vermeulen, 2005; Ratyńska and Szwed, 1996; Szwed and Perkiewicz, 2010; Szwed et al., 1999).

Roads constitute passage routes for plants and they facilitate penetration into the forest for alien species both geographically and ecologically (Andres, 2005; Hansen and Clevenger, 2005; Szwed and Perkiewicz, 2010; Szwed et al., 1999). Impatiens parviflora DC can be given as an example of such neophyte. Roads are also the centres of invasion of those species; particularly those are the sites with replaced soil cover (Brzeg, 1989; Falińska, 2004; Faliński, 1995; Gibert, 1996; Kujawa-Pawlaczyk, 1991; Paszek and Załuski, 2000; Ziarnek, 2000).

A greater number of plant species is encountered along the roads rather than inside a forest. However, the proportion between the forest and non-forest species is disturbed to the advantage of the latter (a forest edge, grassland, clearance, meadow, segetal and ruderal, less frequently swamp). The forest boundary zone is characterized by an increased share of the annual plants which belong to anthropophytes and heliophilous species and occasionally halophytes with the simultaneously reduced importance of sciophilous and forest plants (Balcerkiewicz and Kasprowicz, 1989). The changes may also be observed in the vertical structure of the forest community.

The changes in the species and the structural species changes result from the modification in light conditions and moisture conditions, a specific microrelief, the disturbance of the soil cover and the use of substrate for road construction brought from other locations, sow grass or planting trees and shrubs (Czępińska-Kamińska et al., 1997; Szwed and Perkiewicz, 2010; Szwed et al., 1999). Ziarnek (2000), calculated on the basis of the example of the "Puszcza Bukowa" Forest that among the reported 423 plant species $16 \%$ are never found next to the roads, $47 \%$ taxa grow both near the roads and in the middle of the forests, while $37 \%$ are found only in the vicinity of the roads. Similar values were reported by Paszek and Załuski (1998) from the Górznieńsko-Lidzbarski Landscape Park. Microhabitats formed in relation to the network of roads and paths constitute in the managed even-aged stands the substitutes of small water bodies, watercourses, gaps and openings that are rarely found there and which are typical of the uneven-aged and terminal forest phases. Forest roads contribute to an increased incidence not only of alien species - non-forest, but also of forest even those threatened (Ziarnek, 2000). Moreover, at the boundaries of the sub-Atlantic forest complexes they may comprise thermophilic species of the subcontinental range (Balcerkiewicz and Brzeg, 1993; Balcerkiewicz and Kasprowicz, 1989). Roads are thus a factor enhancing biodiversity (Paszek and Załuski, 2000; Szwed and Perkiewicz, 2010).

The roadside sections of transportation routes are refuges for semi-natural communities in the strongly transformed areas. They promote an increased biodiversity of the region and they constitute the ecological corridors. On the roadsides in the Wielkopolska region, 358 vascular plant species from 225 genera and 65 families including 59 typical forest species and 9 species protected in Poland were inventoried (Szwed and Perkiewicz, 2010). The families Asteraceae and Poaceae were represented by the greatest number of species. The biggest number of plant species was characteristic of the meadow communities Molinio-Arrhenatheretea and the field communities Stellarietea mediae. The representatives of native flora accounted for $72 \%$ while of alien species accounted for $28 \%$.

The main aim of this study was to assess the effect of a road with a very low traffic on the richness of plant species which are present in the neighborhood of the road belt of land in the conditions of pine stands with low trophic status growing on the inland dunes. An additional aim was to identify the role of the road network for this type of habitat as a potential migration corridor for the spread of the invasive species.

\section{METHODS}

The results presented in this paper were obtained from the extensive research conducted to describe the impact of the provincial road no. 150 (DW150) on the neighbouring pine stands in "Puszcza Notecka", the results of which are gradually being prepared for the publication (among others Mizera and Grajewski, 2016).

The field observations were conducted in July-August 2010 in a roadside belt and in the area of roadside stands of the older age classes neighbouring the provincial road DW 150 - from $\mathrm{km} \mathrm{21+220} \mathrm{to} \mathrm{km} \mathrm{26+696}$ of that road. The study was conducted on a set which was perpendicular to the road and which consisted of 
twelve-belt transects 30 or 50 meters wide. Each of them consisted of $3-4$, ten meters plots between which 10 -meters buffer zone not covered by the observations was retained. The location, the way of setting and characteristics of transects together with the designated to them research plots were described in detail by Mizera and Grajewski (2016).

The occurrence and the degree of cover by the individual plant strata, as well as the number of herbaceous plant species, mosses and lichens in accordance with the Braun-Blanquet scale were determined for each plot (Fukarek, 1967; Wysocki and Sikorski, 2009). Data from relevés were listed in the ordered tables presenting the number of plant species in the decreasing order of occurrence for each vegetation layer (providing the percentage of layer cover). The average cover for the individual layers and the mean number of species forming them were calculated based on the tables (Czępińska-Kamińska et al., 1997; Fukarek, 1967; Wysocki and Sikorski, 2009). The nomenclature was adopted after Rutkowski (2011), while that of mosses and lichens after Wójciak (2003). Lichens Lichenes were identified to genera. No detailed identification was conducted within the genus Rubus $\mathrm{L}$. The only division was only for blackberries $R$. species div. and red raspberry $R$. idaeus L. Subspecies were not included in the analyses.
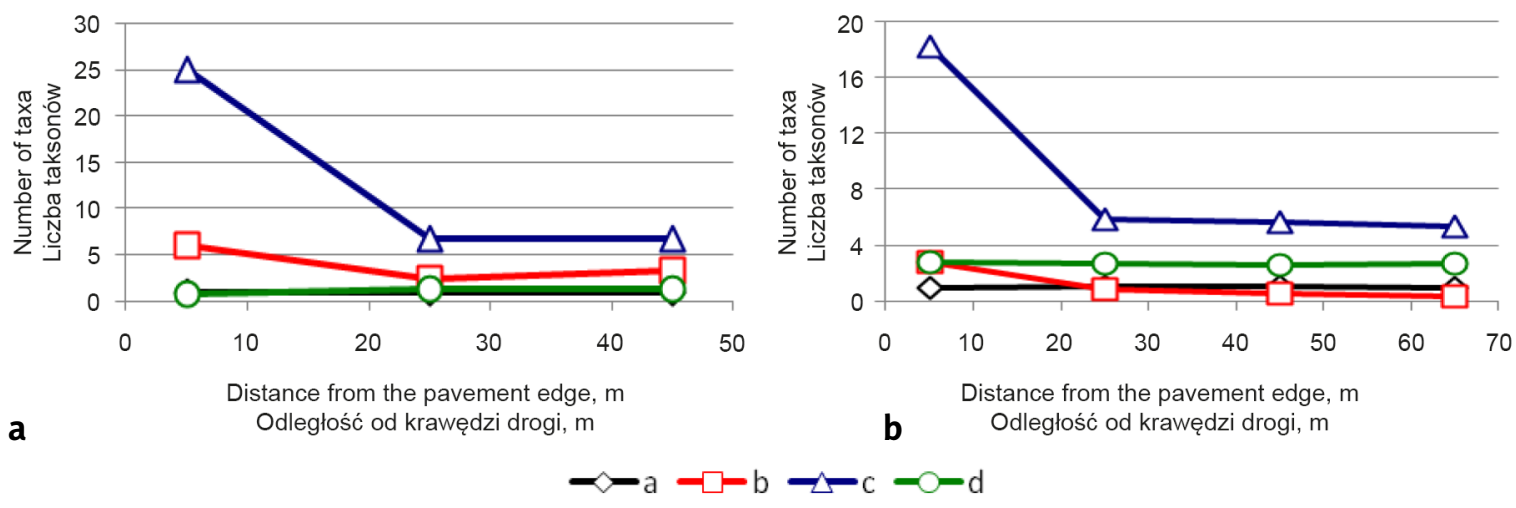

Fig. 1. Changes in mean numbers of taxa forming individual forest vegetation layers $(\mathrm{a}-$ trees, $\mathrm{b}-\mathrm{shrubs}, \mathrm{c}-\mathrm{her}$ baceous, $\mathrm{d}$ - lichen-moss) depending on the distance from the pavement edge (a) in fresh coniferous forest, (b) in fresh mixed coniferous forest

Rys. 1. Zmiany w średnich liczebnościach taksonów tworzących poszczególne warstwy roślinności leśnej (a drzew, b - krzewów, c - zielnej, d - porostowo-mszystej) w zależności od odległości od krawędzi jezdni (a) w borze świeżym, (b) w borze mieszanym świeżym 
Mizera, P., Grajewski, S. M., Kasztelan, A. (2016). The floral characteristics of the road edge effect on the example of a road with low traffic volume in the "Puszcza Notecka" Forest. Acta Sci. Pol. Silv. Colendar. Ratio Ind. Lignar., 15(4), 297-312. DOI: 10.17306/J. AFW.2016.4.30

Table 1. List of taxa recorded in experimental transects in plots no. 1 including ground cover and their abundance in Braun-Blanquet's scale

Tabela 1. Wykaz taksonów odnotowanych na transektach badawczych na działkach nr 1 wraz ze stopniem pokrycia i ich liczebnością w skali Braun-Blanqueta

\begin{tabular}{|c|c|c|c|c|c|c|c|c|c|c|c|c|}
\hline $\begin{array}{l}\text { Number of transect and plot } \\
\text { Numer transektu i działki }\end{array}$ & 1.1 & 2.1 & 3.1 & 4.1 & 5.1 & 6.1 & 7.1 & 8.1 & 9.1 & 10.1 & 11.1 & 12.1 \\
\hline \multicolumn{13}{|c|}{$\mathrm{a}-$ tree layer $-\mathrm{a}-$ warstwa drzew } \\
\hline $\begin{array}{l}\text { Level of coverage, } \% \\
\text { Stopień pokrycia, } \%\end{array}$ & 80 & 40 & 40 & 90 & 80 & 20 & 5 & 20 & 0 & 25 & 15 & 0 \\
\hline $\begin{array}{l}\text { Number of species } \\
\text { Liczba gatunków }\end{array}$ & 1 & 1 & 1 & 1 & 1 & 2 & 1 & 2 & 0 & 1 & 1 & 0 \\
\hline Pinus sylvestris & 5 & 3 & 5 & 5 & 3 & 2 & 1 & 2 & . & 2 & 2 & . \\
\hline Betula pendula & . & . & . & . & . & 2 & . & 2 & . & . & . & . \\
\hline \multicolumn{13}{|c|}{$\mathrm{b}-$ shrub layer $-\mathrm{b}$ - warstwa podszytu } \\
\hline $\begin{array}{l}\text { Level of coverage, } \% \\
\text { Stopień pokrycia, \% }\end{array}$ & 70 & 90 & 60 & 80 & 55 & 40 & 40 & 10 & 50 & 25 & 0 & 50 \\
\hline $\begin{array}{l}\text { Number of species } \\
\text { Liczba gatunków }\end{array}$ & 9 & 3 & 4 & 4 & 11 & 1 & 3 & 4 & 1 & 2 & 0 & 1 \\
\hline Pinus sylvestris & . & . & . & 4 & 1 & 3 & 3 & 2 & 4 & 3 & . & 4 \\
\hline Betula pendula & $\mathrm{r}$ & . & . & $\mathrm{r}$ & 2 & . & r & + & . & $\mathrm{r}$ & . & . \\
\hline Prunus serotina & + & 3 & 1 & . & $\mathrm{r}$ & . & $\mathrm{r}$ & $\mathrm{r}$ & . & . & . & . \\
\hline Robinia pseudoacacia & 1 & 4 & 4 & 3 & 1 & . & . & . & . & . & . & . \\
\hline Acer platanoides & 2 & $\mathrm{r}$ & . & . & $\mathrm{r}$ & . & . & . & . & . & . & . \\
\hline Acer pseudoplatanus & 3 & . & . & $\mathrm{r}$ & + & . & . & . & . & . & . & . \\
\hline Quercus robur & 3 & . & . & . & 3 & . & . & . & . & . & . & . \\
\hline Prunus spinosa & . & . & $\mathrm{r}$ & . & $\mathrm{r}$ & . & . & . & . & . & . & . \\
\hline Pyrus communis & $\mathrm{r}$ & . & . & . & $\mathrm{r}$ & . & . & . & . & . & . & . \\
\hline Juniperus communis & . & . & . & . & . & . & . & 1 & . & . & . & . \\
\hline Caragana arborescens & . & . & $\mathrm{r}$ & . & . & . & . & . & . & . & . & . \\
\hline Malus domestica & . & . & . & . & r & . & . & . & . & . & . & . \\
\hline Prunus padus & . & . & . & . & $\mathrm{r}$ & . & . & . & . & . & . & . \\
\hline Acer negundo & $\mathrm{r}$ & . & . & . & . & . & . & . & . & . & . & . \\
\hline Sambucus racemosa & r & . & . & . & . & . & . & . & . & . & . & . \\
\hline \multicolumn{13}{|c|}{$\mathrm{c}-$ herbaceous layer $-\mathrm{c}-$ warstwa zielna } \\
\hline $\begin{array}{l}\text { Level of coverage, } \% \\
\text { Stopień pokrycia, \% }\end{array}$ & 100 & 90 & 100 & 30 & 90 & 50 & 100 & 100 & 70 & 70 & 50 & 60 \\
\hline $\begin{array}{l}\text { Number of species } \\
\text { Liczba gatunków }\end{array}$ & 19 & 18 & 23 & 18 & 34 & 14 & 22 & 15 & 17 & 18 & 20 & 20 \\
\hline
\end{tabular}


Mizera, P., Grajewski, S. M., Kasztelan, A. (2016). The floral characteristics of the road edge effect on the example of a road with low traffic volume in the "Puszcza Notecka" Forest. Acta Sci. Pol. Silv. Colendar. Ratio Ind. Lignar., 15(4), 297-312. DOI: 10.17306/J. AFW.2016.4.30

Table 1 - cont. / Tabela 1 - cd.

\begin{tabular}{|c|c|c|c|c|c|c|c|c|c|c|c|c|}
\hline $\begin{array}{l}\text { Number of transect and plot } \\
\text { Numer transektu i działki }\end{array}$ & 1.1 & 2.1 & 3.1 & 4.1 & 5.1 & 6.1 & 7.1 & 8.1 & 9.1 & 10.1 & 11.1 & 12.1 \\
\hline Euphorbia cyparissias & 1 & . & . & 2 & 1 & 1 & $\mathrm{r}$ & 2 & $\mathrm{r}$ & 1 & 1 & + \\
\hline Quercus robur & . & . & $\mathrm{r}$ & + & . & + & $\mathrm{r}$ & . & + & + & $\mathrm{r}$ & + \\
\hline Achillea millefolium & + & . & . & + & 1 & . & 2 & + & . & + & + & 1 \\
\hline Hieracium pilosella & . & . & . & + & . & 2 & $\mathrm{r}$ & . & 1 & 1 & + & 1 \\
\hline Agrostis capillaris & . & . & . & 1 & . & . & 1 & 1 & + & 1 & 1 & + \\
\hline Calamagrostis epigejos & 3 & . & . & . & . & . & 3 & 4 & 3 & + & + & + \\
\hline Festuca rubra & . & . & . & + & . & 1 & 2 & . & + & + & 1 & 1 \\
\hline Potentilla reptans & . & + & 1 & + & 1 & . & 1 & + & . & . & . & . \\
\hline Urtica dioica & 2 & 2 & 4 & . & 1 & . & $\mathrm{r}$ & . & . & . & . & . \\
\hline Rumex acetosella & . & . & . & . & . & + & $\mathrm{r}$ & . & $\mathrm{r}$ & . & $\mathrm{r}$ & $\mathrm{r}$ \\
\hline Potentilla arenaria & . & . & . & . & . & 2 & . & . & 1 & 1 & + & 1 \\
\hline Hypericum perforatum & . & . & . & . & . & $\mathrm{r}$ & $\mathrm{r}$ & 1 & $\mathrm{r}$ & . & . & $\mathrm{r}$ \\
\hline Calluna vulgaris & . & . & . & . & . & 1 & . & . & 2 & 3 & 1 & 3 \\
\hline Vaccinium vitis-idaea & . & . & . & . & . & 1 & 1 & 2 & . & 1 & 1 & . \\
\hline Galium verum & + & . & . & . & + & . & $\mathrm{r}$ & $\mathrm{r}$ & $\mathrm{r}$ & . & . & . \\
\hline Plantago lanceolata & $\mathrm{r}$ & . & . & . & $\mathrm{r}$ & $\mathrm{r}$ & $\cdot$ & $\cdot$ & $\mathrm{r}$ & . & + & . \\
\hline Melampyrum pratense & . & . & . & 2 & . & . & $\mathrm{r}$ & + & + & $\mathrm{r}$ & . & . \\
\hline Artemisia vulgaris & + & + & + & + & 1 & . & . & . & . & . & . & . \\
\hline Carex hirta & . & . & . & + & . & 1 & 2 & . & + & . & . & 1 \\
\hline Prunus serotina & . & . & . & . & $\mathrm{r}$ & . & & . & $\mathrm{r}$ & $\mathrm{r}$ & . & $\mathrm{r}$ \\
\hline Rubus sp. div. & 4 & . & 1 & . & . & . & + & 1 & . & . & . & . \\
\hline Geranium robertianum & + & 1 & 1 & . & 1 & . & . & . & . & . & . & . \\
\hline Chaerophyllum temulentum & + & 1 & 2 & . & . & . & . & $\mathrm{r}$ & . & . & . & . \\
\hline Centaurea stoebe & . & . & . & . & . & . & $\mathrm{r}$ & . & $\mathrm{r}$ & . & + & $\mathrm{r}$ \\
\hline Corynephorus canescens & . & . & . & . & . & . & & . & 3 & 3 & 3 & 1 \\
\hline Equisetum arvense & . & + & . & . & . & . & . & . & . & + & . & $\mathrm{r}$ \\
\hline Dryopteris carthusiana & $\mathrm{r}$ & $\mathrm{r}$ & . & . & + & . & . & . & . & . & . & . \\
\hline Chelidonium majus & 2 & 4 & 1 & . & . & . & . & . & . & . & . & . \\
\hline Alliaria petiolata & + & + & 1 & . & . & . & . & . & . & . & . & . \\
\hline Fragaria vesca & . & . & $\mathrm{r}$ & 2 & . & . & . & 1 & . & . & . & . \\
\hline Trifolium repens & . & . & + & + & . & . & $\mathrm{r}$ & . & . & . & . & . \\
\hline Orthilia secunda & + & . & . & + & 1 & . & . & . & . & . & . & . \\
\hline Plantago major & $\mathrm{r}$ & . & + & . & + & . & $\cdot$ & $\cdot$ & . & . & . & . \\
\hline
\end{tabular}


Mizera, P., Grajewski, S. M., Kasztelan, A. (2016). The floral characteristics of the road edge effect on the example of a road with low traffic volume in the "Puszcza Notecka" Forest. Acta Sci. Pol. Silv. Colendar. Ratio Ind. Lignar., 15(4), 297-312. DOI: 10.17306/J. AFW.2016.4.30

Table 1 - cont. / Tabela 1 - cd.

\begin{tabular}{|c|c|c|c|c|c|c|c|c|c|c|c|c|}
\hline $\begin{array}{l}\text { Number of transect and plot } \\
\text { Numer transektu i działki }\end{array}$ & 1.1 & 2.1 & 3.1 & 4.1 & 5.1 & 6.1 & 7.1 & 8.1 & 9.1 & 10.1 & 11.1 & 12.1 \\
\hline Galeopsis tetrahit & $\mathrm{r}$ & + & . & . & + & . & . & . & . & . & . & . \\
\hline Veronica chamaedrys & . & . & + & . & 1 & . & $\mathrm{r}$ & . & . & . & . & . \\
\hline Cirsium arvense & . & $\mathrm{r}$ & $\mathrm{r}$ & . & $\mathrm{r}$ & . & . & . & . & . & . & . \\
\hline Teraxacum officinale & . & . & $\mathrm{r}$ & . & $\mathrm{r}$ & . & $\mathrm{r}$ & . & . & . & . & . \\
\hline Deschampsia flexuosa & . & . & . & . & . & . & . & . & . & + & $\mathrm{r}$ & + \\
\hline Pinus sylvestris & . & . & . & . & . & . & . & $\mathrm{r}$ & . & + & . & . \\
\hline Rumex acetosa & $\mathrm{r}$ & . & . & . & + & . & . & . & . & . & . & . \\
\hline Geum urbanum & . & 1 & + & . & . & . & . & . & . & . & . & . \\
\hline Medicago lupulina & . & . & . & + & . & . & . & . & . & . & . & $\mathrm{r}$ \\
\hline Impatiens parviflora & . & 3 & . & . & 1 & . & . & . & . & . & . & . \\
\hline Galium aparine & . & 1 & + & . & . & . & . & . & . & . & . & . \\
\hline Convolvulus arvensis & . & . & + & . & + & . & . & . & . & . & . & . \\
\hline Glechoma hederacea & . & + & . & . & 1 & . & . & . & . & . & . & . \\
\hline Leontodon hispidus & . & . & . & . & . & . & . & . & . & . & + & + \\
\hline Quercus rubra & . & . & . & + & . & . & . & . & . & . & . & $\mathrm{r}$ \\
\hline Acer platanoides & . & $\mathrm{r}$ & . & . & $\mathrm{r}$ & . & . & . & . & . & . & . \\
\hline Dryopteris filix-mas & . & . & . & . & $\mathrm{r}$ & . & . & . & . & . & . & . \\
\hline Malva neglecta & . & . & . & . & . & . & . & $\mathrm{r}$ & . & . & . & . \\
\hline Chenopodium album & . & . & $\mathrm{r}$ & . & . & . & . & . & . & . & . & . \\
\hline Polygonum arviculare & . & . & $\mathrm{r}$ & . & . & . & . & . & . & . & . & . \\
\hline Rumex crispus & . & 1 & . & . & . & . & . & . & . & . & . & . \\
\hline Armeria maritima & . & . & . & . & . & 1 & . & . & . & . & . & . \\
\hline Silene latifolia & $\mathrm{r}$ & . & . & . & . & . & . & . & . & . & . & . \\
\hline Stellaria media & . & . & $\mathrm{r}$ & . & . & . & . & . & . & . & . & . \\
\hline Ranunculus acris & . & . & . & . & $\mathrm{r}$ & . & . & . & . & . & . & . \\
\hline Sedum acre & . & . & . & . & . & . & . & . & . & . & + & . \\
\hline Prunus padus & . & . & . & . & $\mathrm{r}$ & . & . & . & . & . & . & . \\
\hline Pyrus communis & . & . & . & . & . & . & . & . & . & $\mathrm{r}$ & . & . \\
\hline Rosa canina & . & . & . & . & $\mathrm{r}$ & . & . & . & . & . & . & . \\
\hline Rubus idaeus & . & . & . & . & 1 & . & . & . & . & . & . & . \\
\hline Lotus corniculatus & . & . & . & . & + & . & . & . & . & . & . & . \\
\hline Trifolium arvense & . & . & . & . & . & . & . & . & . & . & $\mathrm{r}$ & . \\
\hline Trifolium pratense & . & . & . & . & + & . & . & . & . & . & . & . \\
\hline Daucus carota & . & . & . & . & $\mathrm{r}$ & . & . & . & . & . & . & . \\
\hline
\end{tabular}


Mizera, P., Grajewski, S. M., Kasztelan, A. (2016). The floral characteristics of the road edge effect on the example of a road with low traffic volume in the "Puszcza Notecka" Forest. Acta Sci. Pol. Silv. Colendar. Ratio Ind. Lignar., 15(4), 297-312. DOI: 10.17306/J. AFW.2016.4.30

Table 1 - cont. / Tabela 1 - cd.

\begin{tabular}{|c|c|c|c|c|c|c|c|c|c|c|c|c|}
\hline $\begin{array}{l}\text { Number of transect and plot } \\
\text { Numer transektu i działki }\end{array}$ & 1.1 & 2.1 & 3.1 & 4.1 & 5.1 & 6.1 & 7.1 & 8.1 & 9.1 & 10.1 & 11.1 & 12.1 \\
\hline Heracleum shpondylium & . & $\mathrm{r}$ & . & . & . & . & . & . & . & . & . & . \\
\hline Vaccinium myrtillus & . & . & . & . & . & . & . & . & . & 1 & . & . \\
\hline Galium mollugo & . & . & . & . & . & . & $\mathrm{r}$ & . & . & . & . & . \\
\hline Ajuga reptans & . & . & . & . & . & . & . & + & . & . & . & . \\
\hline Thymus serpyllum & . & . & . & + & . & . & . & . & . & . & . & . \\
\hline Knautia arvensis & . & . & $\mathrm{r}$ & . & . & . & . & . & . & . & . & . \\
\hline Jasione montana & . & . & . & . & . & . & . & . & . & . & + & . \\
\hline Conyza canadensis & . & . & . & . & $\mathrm{r}$ & . & . & . & . & . & . & . \\
\hline Erigeron acer & . & . & . & . & . & . & . & . & . & . & $\mathrm{r}$ & . \\
\hline Helichrysum arenarium & . & . & . & . & . & 1 & . & . & . & . & . & . \\
\hline Mycelis muralis & . & . & . & . & 1 & . & . & . & . & . & . & . \\
\hline Fagus sylvatica & . & . & . & $\mathrm{r}$ & . & . & . & . & . & . & . & . \\
\hline Ulmus laevis & $\mathrm{r}$ & . & . & . & . & . & . & . & . & . & . & . \\
\hline Acer pseudoplatanus & . & . & . & . & + & . & . & . & . & . & . & . \\
\hline Frangula alnus & . & . & . & . & . & . & $\mathrm{r}$ & . & . & . & . & . \\
\hline Polygonatum multiflorum & . & . & . & . & . & $\mathrm{r}$ & . & . & . & . & . & . \\
\hline Anthoxanthum odoratum & . & . & . & + & . & . & . & . & . & . & . & . \\
\hline Bromus sterilis & . & . & $\mathrm{r}$ & . & . & . & . & . & . & . & . & . \\
\hline Dactylis glomerata & . & . & . & . & $\mathrm{r}$ & . & . & . & . & . & . & . \\
\hline Deschampsia caespitosa & . & . & . & . & 4 & . & . & . & . & . & . & . \\
\hline \multicolumn{13}{|c|}{$\mathrm{d}$ - lichen-moss layer - $\mathrm{d}$ - warstwa porostowo-mszysta } \\
\hline $\begin{array}{l}\text { Level of coverage, \% } \\
\text { Stopien pokrycia, \% }\end{array}$ & 50 & 0 & 0 & 90 & 70 & 50 & 70 & 90 & 60 & 90 & 20 & 95 \\
\hline $\begin{array}{l}\text { Number of species } \\
\text { Liczba gatunków }\end{array}$ & 1 & 0 & 0 & 2 & 2 & 2 & 4 & 3 & 3 & 5 & 4 & 3 \\
\hline Pleurozium schreberi & 4 & . & . & 4 & 4 & 3 & 4 & 5 & 4 & 5 & 2 & 5 \\
\hline Cladonia sp. div. & . & . & . & 3 & . & 2 & + & + & + & + & 1 & + \\
\hline Dicranum polysetum & . & . & . & . & . & . & + & + & + & + & 1 & + \\
\hline Peltigera canina & . & . & . & . & . & . & + & . & . & . & . & . \\
\hline Hylocomnium splendens & . & . & . & . & 2 & . & . & . & . & . & . & . \\
\hline Hypnum cupressiforme & . & . & . & . & . & . & . & . & . & + & . & . \\
\hline Pohlia nutans & . & . & . & . & . & . & . & . & . & . & + & . \\
\hline Polytrichum formosum & . & . & . & . & . & . & . & . & . & + & . & \\
\hline
\end{tabular}


Mizera, P., Grajewski, S. M., Kasztelan, A. (2016). The floral characteristics of the road edge effect on the example of a road with low traffic volume in the "Puszcza Notecka" Forest. Acta Sci. Pol. Silv. Colendar. Ratio Ind. Lignar., 15(4), 297-312. DOI: 10.17306/J. AFW.2016.4.30

Table 2. List of taxa recorded in experimental transects in plots no. 2 including ground cover and their abundance in Braun-Blanquet's scale

Tabela 2. Wykaz taksonów odnotowanych na transektach badawczych na działkach nr 2 wraz ze stopniem pokrycia i ich liczebnością w skali Braun-Blanqueta

\begin{tabular}{|c|c|c|c|c|c|c|c|c|c|c|c|c|}
\hline $\begin{array}{l}\text { Number of transect and plot } \\
\text { Numer transektu i działki }\end{array}$ & 1.2 & 2.2 & 3.2 & 4.2 & 5.2 & 6.2 & 7.2 & 8.2 & 9.2 & 10.2 & 11.2 & 12.2 \\
\hline \multicolumn{13}{|c|}{$\mathrm{a}-$ tree layer $-\mathrm{a}-$ warstwa drzew } \\
\hline $\begin{array}{l}\text { Level of coverage, } \% \\
\text { Stopień pokrycia, } \%\end{array}$ & 80 & 80 & 90 & 90 & 95 & 80 & 90 & 80 & 60 & 80 & 70 & 75 \\
\hline $\begin{array}{l}\text { Number of species } \\
\text { Liczba gatunków }\end{array}$ & 1 & 1 & 1 & 2 & 1 & 1 & 1 & 1 & 1 & 1 & 1 & 1 \\
\hline Pinus sylvestris & 5 & 5 & 5 & 5 & 5 & 5 & 5 & 5 & 4 & 5 & 4 & 5 \\
\hline Betula pendula & . & . & . & $\mathrm{r}$ & . & . & . & . & . & . & . & . \\
\hline \multicolumn{13}{|c|}{$\mathrm{b}-$ shrub layer $-\mathrm{b}-$ warstwa podszytu } \\
\hline $\begin{array}{l}\text { Level of coverage, } \% \\
\text { Stopień pokrycia, \% }\end{array}$ & 0 & 80 & 100 & 0 & 10 & 30 & 50 & 30 & 0 & 0 & 0 & 0 \\
\hline $\begin{array}{l}\text { Number of species } \\
\text { Liczba gatunków }\end{array}$ & 0 & 3 & 2 & 0 & 2 & 1 & 3 & 4 & 0 & 0 & 0 & 0 \\
\hline Pinus sylvestris & . & . & . & . & . & 3 & 4 & 3 & . & . & . & . \\
\hline Betula pendula & . & . & . & . & 2 & . & $\mathrm{r}$ & 2 & . & . & . & . \\
\hline Prunus serotina & . & 2 & 5 & . & . & . & . & + & . & . & . & . \\
\hline Robinia pseudoacacia & . & 4 & 1 & . & . & . & . & . & . & . & . & . \\
\hline Juniperus communis & . & . & . & . & . & . & . & + & . & . & . & . \\
\hline Fagus sylvatica & . & . & . & . & . & . & $\mathrm{r}$ & . & . & . & . & . \\
\hline Quercus robur & . & . & . & . & $\mathrm{r}$ & . & . & . & . & . & . & . \\
\hline Frangula alnus & . & r & . & . & . & . & . & . & . & . & . & . \\
\hline \multicolumn{13}{|c|}{$\mathrm{c}-$ herbaceous layer $-\mathrm{c}-$ warstwa zielna } \\
\hline $\begin{array}{l}\text { Level of coverage, \% } \\
\text { Stopień pokrycia, \% }\end{array}$ & 10 & 90 & 30 & 10 & 20 & 25 & 90 & 80 & 60 & 50 & 10 & 60 \\
\hline $\begin{array}{l}\text { Number of species } \\
\text { Liczba gatunków }\end{array}$ & 2 & 7 & 4 & 3 & 9 & 4 & 8 & 11 & 8 & 6 & 6 & 5 \\
\hline Quercus robur & 2 & . & . & 1 & 2 & + & + & $\mathrm{r}$ & + & . & + & + \\
\hline Calluna vulgaris & . & . & . & . & . & + & 2 & + & 3 & 3 & 1 & + \\
\hline Vaccinium vitis-idaea & . & . & . & . & . & 2 & 3 & 4 & 2 & 2 & 2 & 4 \\
\hline Deschampsia flexuosa & 1 & . & . & . & . & . & 2 & 2 & 1 & $\mathrm{r}$ & + & + \\
\hline Vaccinium myrtillus & . & . & . & . & + & . & 2 & 2 & 1 & . & 1 & . \\
\hline Pinus sylvestris & . & . & . & . & + & . & . & . & + & . & + & 2 \\
\hline Sorbus aucuparia & . & $\mathrm{r}$ & $\mathrm{r}$ & . & . & . & . & . & $\mathrm{r}$ & $\mathrm{r}$ & . & . \\
\hline Melampyrum pratense & . & . & . & 2 & . & 1 & + & 1 & . & . & . & . \\
\hline Betula pendula & . & . & . & . & + & . & + & + & . & $\mathrm{r}$ & . & . \\
\hline Festuca rubra & . & . & . & . & 1 & . & 1 & + & + & . & . & . \\
\hline Dryopteris carthusiana & . & + & $\mathrm{r}$ & . & 1 & . & . & . & . & . & . & . \\
\hline Prunus serotina & . & $\mathrm{r}$ & . & . & + & . & . & $\mathrm{r}$ & . & . & . & . \\
\hline Quercus rubra & . & . & . & 1 & . & . & . & $\mathrm{r}$ & . & $\mathrm{r}$ & . & . \\
\hline Impatiens parviflora & . & 5 & 3 & . & . & . & . & . & . & . & . & . \\
\hline
\end{tabular}


Mizera, P., Grajewski, S. M., Kasztelan, A. (2016). The floral characteristics of the road edge effect on the example of a road with low traffic volume in the "Puszcza Notecka" Forest. Acta Sci. Pol. Silv. Colendar. Ratio Ind. Lignar., 15(4), 297-312. DOI: 10.17306/J. AFW.2016.4.30

Table 2 - cont. / Tabela 2 - cd.

\begin{tabular}{|c|c|c|c|c|c|c|c|c|c|c|c|c|}
\hline $\begin{array}{l}\text { Number of transect and plot } \\
\text { Numer transektu i działki }\end{array}$ & 1.2 & 2.2 & 3.2 & 4.2 & 5.2 & 6.2 & 7.2 & 8.2 & 9.2 & 10.2 & 11.2 & 12.2 \\
\hline Fagus sylvatica & . & $\mathrm{r}$ & . & . & $\mathrm{r}$ & . & . & . & . & . & . & . \\
\hline Pteridium aquilinum & . & . & . & . & . & . & . & 1 & . & . & . & . \\
\hline Rumex acetosella & . & . & . & . & + & . & . & . & . & . & . & . \\
\hline Moehringia trinervia & . & 1 & . & . & . & . & . & . & . & . & . & . \\
\hline Chelidonium majus & . & + & . & . & . & . & . & . & . & . & . & . \\
\hline Frangula alnus & . & . & $\mathrm{r}$ & . & . & . & . & . & . & . & . & . \\
\hline \multicolumn{13}{|c|}{$\mathrm{d}$ - lichen-moss layer $-\mathrm{d}$ - warstwa porostowo-mszysta } \\
\hline $\begin{array}{l}\text { Level of coverage, \% } \\
\text { Stopień pokrycia, \% }\end{array}$ & 100 & 0 & 0 & 100 & 100 & 100 & 100 & 100 & 100 & 100 & 100 & 100 \\
\hline $\begin{array}{l}\text { Number of species } \\
\text { Liczba gatunków }\end{array}$ & 2 & 0 & 0 & 3 & 4 & 3 & 2 & 2 & 2 & 3 & 4 & 3 \\
\hline Pleurozium schreberi & 5 & . & . & 5 & 5 & 5 & 5 & 5 & 5 & 5 & 5 & 5 \\
\hline Dicranum polysetum & + & . & . & + & 1 & + & + & + & . & + & + & + \\
\hline Cladonia sp. div. & . & . & . & 2 & . & + & . & . & + & + & + & + \\
\hline Hypnum cupressiforme & . & . & . & . & . & . & . & . & . & . & + & . \\
\hline Leucobryum glaucum & . & . & . & . & 2 & . & . & . & . & . & . & . \\
\hline Polytrichum formosum & . & . & . & . & 2 & . & . & . & . & . & . & . \\
\hline
\end{tabular}

Table 3. List of taxa recorded in experimental transects in plots no. 3 including ground cover and their abundance in Braun-Blanquet's scale

Tabela 3. Wykaz taksonów odnotowanych na transektach badawczych na działkach $\mathrm{nr} 3$ wraz ze stopniem pokrycia i ich liczebnością w skali Braun-Blanqueta

\begin{tabular}{|c|c|c|c|c|c|c|c|c|c|c|c|c|}
\hline $\begin{array}{l}\text { Number of transect and plot } \\
\text { Numer transektu i działki }\end{array}$ & 1.3 & 2.3 & 3.3 & 4.3 & 5.3 & 6.3 & 7.3 & 8.3 & 9.3 & 10.3 & 11.3 & 12.3 \\
\hline \multicolumn{13}{|c|}{$\mathrm{a}-$ tree layer $-\mathrm{a}-$ warstwa drzew } \\
\hline $\begin{array}{l}\text { Level of coverage, } \% \\
\text { Stopień pokrycia, } \%\end{array}$ & 70 & 70 & 70 & 80 & 90 & 70 & 90 & 60 & 80 & 70 & 80 & 70 \\
\hline $\begin{array}{l}\text { Number of species } \\
\text { Liczba gatunków }\end{array}$ & 1 & 1 & 1 & 1 & 1 & 1 & 1 & 2 & 1 & 1 & 1 & 1 \\
\hline Pinus sylvestris & 4 & 4 & 4 & 5 & 5 & 4 & 5 & 4 & 5 & 4 & 5 & 4 \\
\hline Betula pendula & . & . & . & . & . & . & . & $\mathrm{r}$ & . & . & . & . \\
\hline \multicolumn{13}{|c|}{$\mathrm{b}$ - shrub layer $-\mathrm{b}$ - warstwa podszytu } \\
\hline $\begin{array}{l}\text { Level of coverage, \% } \\
\text { Stopień pokrycia, \% }\end{array}$ & 0 & 90 & 40 & 0 & 0 & 30 & 10 & 0 & 5 & 0 & 0 & 0 \\
\hline $\begin{array}{l}\text { Number of species } \\
\text { Liczba gatunków }\end{array}$ & 0 & 6 & 4 & 0 & 0 & 1 & 2 & 0 & 2 & 0 & 0 & 0 \\
\hline Pinus sylvestris & . & . & $\mathrm{r}$ & . & . & 3 & 2 & . & 1 & . & . & . \\
\hline Betula pendula & . & 1 & $\mathrm{r}$ & . & . & . & $\mathrm{r}$ & . & . & . & . & . \\
\hline Robinia pseudoacacia & . & 4 & 2 & . & . & . & . & . & . & . & . & . \\
\hline Prunus serotina & . & 3 & 3 & . & . & . & . & . & . & . & . & . \\
\hline
\end{tabular}


Mizera, P., Grajewski, S. M., Kasztelan, A. (2016). The floral characteristics of the road edge effect on the example of a road with low traffic volume in the "Puszcza Notecka" Forest. Acta Sci. Pol. Silv. Colendar. Ratio Ind. Lignar., 15(4), 297-312. DOI: 10.17306/J. AFW.2016.4.30

Table 3 - cont. / Tabela 3 - cd.

\begin{tabular}{|c|c|c|c|c|c|c|c|c|c|c|c|c|}
\hline $\begin{array}{l}\text { Number of transect and plot } \\
\text { Numer transektu i działki }\end{array}$ & 1.3 & 2.3 & 3.3 & 4.3 & 5.3 & 6.3 & 7.3 & 8.3 & 9.3 & 10.3 & 11.3 & 12.3 \\
\hline Juniperus communis & . & . & . & . & . & . & . & . & $\mathrm{r}$ & . & . & . \\
\hline Sorbus aucuparia & . & + & . & . & . & . & . & . & . & . & . & . \\
\hline Acer platanoides & . & + & . & . & . & . & . & . & . & . & . & . \\
\hline Frangula alnus & . & 1 & . & . & . & . & . & . & . & . & . & . \\
\hline \multicolumn{13}{|c|}{$\mathrm{c}-$ herbaceous layer $-\mathrm{c}-$ warstwa zielna } \\
\hline $\begin{array}{l}\text { Level of coverage, } \% \\
\text { Stopień pokrycia, } \%\end{array}$ & 10 & 40 & 40 & 5 & 10 & 25 & 90 & 90 & 70 & 20 & 35 & 50 \\
\hline $\begin{array}{l}\text { Number of species } \\
\text { Liczba gatunków }\end{array}$ & 3 & 8 & 8 & 3 & 4 & 6 & 8 & 5 & 8 & 7 & 6 & 5 \\
\hline Deschampsia flexuosa & 1 & 2 & + & + & . & + & 1 & 2 & + & + & + & 1 \\
\hline Pinus sylvestris & + & . & . & . & . & + & + & . & + & + & + & 1 \\
\hline Vaccinium vitis-idaea & . & . & . & . & . & 2 & 5 & 5 & 3 & 2 & 1 & 3 \\
\hline Quercus robur & 2 & . & + & 1 & 2 & . & . & . & + & $\mathrm{r}$ & $\mathrm{r}$ & . \\
\hline Calluna vulgaris & . & . & . & . & . & 1 & + & 2 & 2 & + & . & + \\
\hline Vaccinium myrtillus & . & . & . & . & . & 2 & 1 & 2 & 1 & . & 3 & 2 \\
\hline Sorbus aucuparia & . & 2 & 1 & . & $\mathrm{r}$ & . & . & . & + & . & $\mathrm{r}$ & . \\
\hline Prunus serotina & . & 2 & 3 & . & . & . & . & . & . & $\mathrm{r}$ & . & . \\
\hline Melampyrum pratense & . & . & . & . & . & + & + & 1 & . & . & . & . \\
\hline Festuca rubra & . & . & + & . & . & . & + & . & + & . & . & . \\
\hline Dryopteris carthusiana & . & 1 & . & . & 1 & . & . & . & . & . & . & . \\
\hline Betula pendula & . & . & . & . & + & . & + & . & . & . & . & . \\
\hline Quercus rubra & . & . & $\mathrm{r}$ & 1 & . & . & . & . & . & . & . & . \\
\hline Frangula alnus & . & 2 & + & . & . & . & . & . & . & . & . & . \\
\hline Rumex acetosella & . & . & . & . & . & . & . & . & . & $\mathrm{r}$ & . & . \\
\hline Hieracium vulgatum & . & $\mathrm{r}$ & . & . & . & . & . & . & . & . & . & . \\
\hline Hieracium pilosella & . & . & $\mathrm{r}$ & . & . & . & . & . & . & . & . & . \\
\hline Convolvulus arvensis & . & $\mathrm{r}$ & . & . & . & . & . & . & . & . & . & . \\
\hline Acer platanoides & . & + & . & . & . & . & . & . & . & . & . & . \\
\hline \multicolumn{13}{|c|}{$\mathrm{d}$ - lichen-moss layer $-\mathrm{d}$ - warstwa porostowo-mszysta } \\
\hline $\begin{array}{l}\text { Level of coverage, } \% \\
\text { Stopień pokrycia, \% }\end{array}$ & 100 & 20 & 90 & 100 & 100 & 100 & 100 & 100 & 100 & 100 & 100 & 100 \\
\hline $\begin{array}{l}\text { Number of species } \\
\text { Liczba gatunków }\end{array}$ & 3 & 1 & 1 & 3 & 2 & 2 & 2 & 3 & 1 & 3 & 4 & 2 \\
\hline Pleurozium schreberi & 5 & 2 & 5 & 5 & 5 & 5 & 5 & 5 & 5 & 5 & 5 & 5 \\
\hline Dicranum polysetum & + & . & . & + & 2 & + & + & + & . & . & + & + \\
\hline Cladonia sp div. & . & . & . & 2 & . & . & . & . & . & + & + & . \\
\hline Leucobryum glaucum & 1 & . & . & . & . & . & . & + & . & . & + & . \\
\hline Polytrichum formosum & . & . & . & . & . & . & . & . & . & + & . & . \\
\hline
\end{tabular}


Mizera, P., Grajewski, S. M., Kasztelan, A. (2016). The floral characteristics of the road edge effect on the example of a road with low traffic volume in the "Puszcza Notecka" Forest. Acta Sci. Pol. Silv. Colendar. Ratio Ind. Lignar., 15(4), 297-312. DOI: 10.17306/J. AFW.2016.4.30

Table 4. List of taxa recorded in experimental transects in plots no. 4 including ground cover and their abundance in Braun-Blanquet's scale

Tabela 4. Wykaz taksonów odnotowanych na transektach badawczych na działkach nr 4 wraz ze stopniem pokrycia i ich liczebnością w skali Braun-Blanqueta

\begin{tabular}{|c|c|c|c|}
\hline $\begin{array}{l}\text { Number of transect and plot } \\
\text { Numer transektu i działki }\end{array}$ & 6.4 & 8.4 & 12.4 \\
\hline \multicolumn{4}{|c|}{$\mathrm{a}-$ tree layer $-\mathrm{a}-$ warstwa drzew } \\
\hline $\begin{array}{l}\text { Level of coverage, \% } \\
\text { Stopień pokrycia, \% }\end{array}$ & 75 & 70 & 80 \\
\hline $\begin{array}{l}\text { Number of species } \\
\text { Liczba gatunków }\end{array}$ & 4 & 3 & 3 \\
\hline Pinus sylvestris & 5 & 4 & 5 \\
\hline \multicolumn{4}{|c|}{$\mathrm{b}-$ shrub layer $-\mathrm{b}-$ warstwa podszytu } \\
\hline $\begin{array}{l}\text { Level of coverage, \% } \\
\text { Stopień pokrycia, \% }\end{array}$ & 15 & 0 & 0 \\
\hline $\begin{array}{l}\text { Number of species } \\
\text { Liczba gatunków }\end{array}$ & 1 & 0 & 0 \\
\hline Pinus sylvestris & 2 & . & . \\
\hline \multicolumn{4}{|c|}{$\mathrm{c}-$ herbaceous layer $-\mathrm{c}-$ warstwa zielna } \\
\hline $\begin{array}{l}\text { Level of coverage, } \% \\
\text { Stopień pokrycia, } \%\end{array}$ & 25 & 90 & 60 \\
\hline $\begin{array}{l}\text { Number of species } \\
\text { Liczba gatunków }\end{array}$ & 4 & 6 & 6 \\
\hline Calluna vulgaris & + & + & $\mathrm{r}$ \\
\hline Vaccinium myrtillus & 2 & 2 & 3 \\
\hline Vaccinium vitis-idaea & 1 & 5 & 2 \\
\hline Pinus sylvestris & + & & $\mathrm{r}$ \\
\hline Deschampsia flexuosa & . & 1 & 1 \\
\hline Melampyrum pratense & . & + & . \\
\hline Quercus robur & . & . & + \\
\hline Festuca rubra & . & + & . \\
\hline \multicolumn{4}{|c|}{$\mathrm{d}$ - lichen-moss layer $-\mathrm{d}-$ warstwa porostowo-mszysta } \\
\hline $\begin{array}{l}\text { Level of coverage, } \% \\
\text { Stopień pokrycia, } \%\end{array}$ & 100 & 100 & 100 \\
\hline $\begin{array}{l}\text { Number of species } \\
\text { Liczba gatunków }\end{array}$ & 4 & 2 & 2 \\
\hline Pleurozium schreberi & 5 & 5 & 5 \\
\hline Dicranum polysetum & + & + & + \\
\hline Leucobryum glaucum & + & . & . \\
\hline Polytrichum formosum & + & . & . \\
\hline
\end{tabular}

All the plant taxa identified in thirty six experimental sites were divided into three groups (Table 5). The first one includes taxa found solely in the immediate vicinity of the road (up to $10 \mathrm{~m}$ from the pavement edge), the second consists of the taxa found both along the road and in the stand interior, the third comprises of taxa found only inside the stand (mix. $10 \mathrm{~m}$ from the pavement edge). The first group comprises of 76 taxa $(70 \%)$, the second of $27(25 \%)$, while the third is composed of only 5 taxa $(5 \%)$. 
Mizera, P., Grajewski, S. M., Kasztelan, A. (2016). The floral characteristics of the road edge effect on the example of a road with low traffic volume in the "Puszcza Notecka" Forest. Acta Sci. Pol. Silv. Colendar. Ratio Ind. Lignar., 15(4), 297-312. DOI: 10.17306/J. AFW.2016.4.30

Table 5. Taxa recognized in all 39 research plots are present: A - only in the immediate vicinity of the road (zone $0-10 \mathrm{~m}$ from the pavement edge), B - found both along the road and in the stand interior, $\mathrm{C}-$ found only inside the stand ( $>10 \mathrm{~m}$ from the pavement edge)

Tabela 5. Taksony rozpoznane na wszystkich 39 działkach badawczych występujące: A - wyłącznie w bezpośrednim sąsiedztwie drogi (strefa 0-10 m od krawędzi nawierzchni), B - zarówno przy drodze, jak i wewnątrz drzewostanu, C - wyłącznie wewnątrz drzewostanu (>10 m od krawędzi nawierzchni)

\begin{tabular}{|c|c|c|}
\hline A & B & $\mathrm{C}$ \\
\hline $\begin{array}{l}\text { Peltigera canina (L.) Wild., Pohlia nutans (Hedw.) Lindb., Hylocom- } \\
\text { nium splendens (Hedw.) B.S.G., Equisetum arvense L., Dryopteris } \\
\text { filix-mas (L.) Schott, Urtica dioica L., Ulmus laevis Pall., Polygonum } \\
\text { arviculare L., Rumex acetosa L., R. crispus L., Chenopodium album } \\
\text { L., Silene latifolia Poiret, Stellaria media (L.) Vill., Ranunculus acris } \\
\text { L., Alliaria petiolata (Bieb.) Cav. et Grande, Sedum acre L., Fraga- } \\
\text { ria vesca L., Geum urbanum L., Malus domestica Borkh., Potentilla } \\
\text { arenaria Borkh., P. reptans L., Prunus padus L., P. spinosa L., Pyrus } \\
\text { communis L. em. Gaertner, Rosa canina L., Rubus idaeus L., Rubus } \\
\text { spp. L., Caragana arborescens Lam., Lotus corniculatus L., Medicago } \\
\text { lupulina L., Trifolium arvense L., T. pratense L., T. repens L., Gera- } \\
\text { nium robertianum L., Euphorbia cyparissias L., Acer negundo L., A. } \\
\text { pseudoplatanus L., Malva neglecta Wallr., Hypericum perforatum L., } \\
\text { Chaerophyllum temulentum L., Daucus carota L., Heracleum sphon- } \\
\text { dylium L., Orthilia secunda (L.) House, Armeria maritima (Miller) } \\
\text { Willd., Galium aparine L., G. mollugo L., G. verum L., Ajuga reptans } \\
\text { L., Galeopsis tetrahit L., Glechoma hederacea L., Thymus serpyllum } \\
\text { L., Veronica chamaedrys L., Plantago lanceolata L., P. major L., Sam- } \\
\text { bucus racemosa L., Knautia arvensis (L.) Coult., Jasione montana L., } \\
\text { Achillea millefolium L., Artemisia vulgaris L., Centaurea stoebe L., } \\
\text { Cirsium arvense (L.) Scop., Conyza canadensis (L.) Cronq., Erigeron } \\
\text { acer L., Helichrysum arenarium (L.) Moench, Leontodon hispidus L., } \\
\text { Mycelis muralis (L.) Dum., Teraxacum officinale Weber, Polygonatum } \\
\text { multiflorum (L.) All., Agrostis capillaris L., Anthoxanthum odoratum } \\
\text { (L.) P.B., Bromus sterilis L., Calamagrostis epigejos (L.) Roth, Co- } \\
\text { rynephorus canescens (L.) P.B., Dactylis glomerata L., Deschampsia } \\
\text { caesnitosa (L.) PB Carex hirta } \text { L }\end{array}$ & $\begin{array}{l}\text { Cladonia P. Browne, Poly- } \\
\text { trichum formosum Hedw., } \\
\text { Dicranum polysetum Sw., } \\
\text { Pleurozium schreberi (Brid.) } \\
\text { Mitt., Hypnum cupressiforme } \\
\text { Hedw., Dryopteris carthu- } \\
\text { siana (Vill.) H.P. Fuchs, } \\
\text { Pinus sylvestris L., Juniperus } \\
\text { communis L., Betula pen- } \\
\text { dula } \text { Roth, Fagus sylvatica } \\
\text { L., Quercus robur L., Q. } \\
\text { rubra L., Rumex acetosella } \\
\text { L., Chelidonium majus L., } \\
\text { Prunus serotina Ehrh., Ro- } \\
\text { binia pseudoacacia L., Acer } \\
\text { platanoides L., Impatiens pa- } \\
\text { rviflora DC., Frangula alnus } \\
\text { Mill., Calluna vulgaris (L.) } \\
\text { Hull, Vaccinium myrtillus L., } \\
\text { V. vitis-idaea L., Convolvulus } \\
\text { arvensis L., Melampyrum } \\
\text { pratense L., Hieracium } \\
\text { pilosella L., Deschampsia } \\
\text { flexuosa (L.) Trin., Festuca } \\
\text { rubra L. }\end{array}$ & $\begin{array}{l}\text { Leucobryum glaucum } \\
\text { (Hedw.) Ångstr., Pte- } \\
\text { ridium aquilinum (L.) } \\
\text { Kuhn, Moehringia } \\
\text { trinervia (L.) Clairv., } \\
\text { Sorbus aucuparia L. } \\
\text { oraz Hieracium vulga- } \\
\text { tum Fries. }\end{array}$ \\
\hline
\end{tabular}

\section{DISCUSSION}

The results of quantitative vegetation analysis are generally consistent with those recorded in the other investigated sections of the Polish roads (Czepińska-Kamińska et al., 1997). The listed differences in the numbers of species in the edge and interior of the stand result from the incorporation of flora growing in roadsides into this study.

When analysing qualitative relationships we need to stress the dominance of taxa whose occurrence is closely related with the vicinity of the road (Marcantonio et al., 2013; Watkins et al., 2001). In the results reported by other researchers (Paszek and Załuski,
1998; Ziarnek, 2000), the most numerous group was composed of species for which the linear gap in the stand, such as a road, is of no importance. Harsher forest site conditions in the "Puszcza Notecka" Forest might have shifted these proportions towards the first of the plant groups. An increase in fertility of the forest site in the roadside zone resulted in a considerable increase in the richness of species. In mesotrophic forest sites of the "Puszcza Bukowa" Forest (Ziarnek, 2000), where the forest floor vegetation was probably formed by many herbaceous plant species, the diversity of roadside flora did not create such a tremendous contrast to the sites located inside the stand. 
Mizera, P., Grajewski, S. M., Kasztelan, A. (2016). The floral characteristics of the road edge effect on the example of a road with low traffic volume in the "Puszcza Notecka" Forest. Acta Sci. Pol. Silv. Colendar. Ratio Ind. Lignar., 15(4), 297-312. DOI: 10.17306/J. AFW.2016.4.30

According to the floristic data collected in the Bialowieża National Park (Faliński, 1961), the percentage of species found both at the roadside and inside the stand in the case of oak-hornbeam forest was as high as $72 \%$ while in the case of fresh coniferous forest it was only $55 \%$. In both cases - in the investigations conducted by the authors of this study and in the cited comparative literature data - the least numerous group consisted of taxa evidently avoiding the vicinity of roads.

Convolvulus arvensis and Hieracium pilosella were found inside the stand once. It occurred in transects established in the fresh mixed coniferous forest site and in the plots located the farthest from the road but at the same time adjacent to water bodies. The occurrence of these species was thus influenced by the open space and for this reason they should rather be classified to the first group and they appeared in the second group accidentally. In the same location, $H$. vulgatum from the third group was found and similarly it should be classified either to the first group or excluded. Generally the occurrence of species from the third group only inside the stand is not confirmed in the view of literary sources on botany (Rutkowski, 2011; Wójciak, 2003). Those species do not avoid open spaces and their absence along road DW 150 could have been accidental.

In this study only one seedling of Frangula alnus was found, in the plot adjacent to the road. The less abundant occurrence of $F$. alnus in the vicinity of a road was also indicated by Czępińska-Kamińska et al. (1997). At the same time, those researchers confirmed the less frequent occurrence of Vaccinium myrtillus, Calluna vulgaris and Pteridium aquilinum in the vicinity of transportation routes and a lack of the influence of roads on the frequency of occurrence of Melampyrum pratense and the more abundant occurrence of Fragaria vesca, singly also Pyrus communis, Acer pseudoplatanus and A. platanoides. In the above mentioned study, the increased occurrence of Urtica dioica and Plantago major was not confirmed while these species were reported along road DW150.

The consistency of observations within this study and those reported in the cited publication was also found for the occurrence of Impatiens parviflora. The expansion of this neophyte in the areas located in the vicinity of transportation routes, described by many other authors, was not observed in either of these studies. Here, we may observe different phases of expansion, i.e. initial in transect no. 5 and final in transects nos. 2 and 3 when this newcomer has found more advantageous conditions inside the forest complex and is retreating from its edge which it used earlier as the migration route. We also need to mention here that the occurrence of I. parviflora in coniferous forest sites is rather a novel finding. The majority of authors ascribe the occurrence of small balsam to fertile and moist deciduous forests. In the Białowieża Forest, it was found solely in oak-hornbeam forests (Kujawa-Pawlaczyk, 1991). This may indicate an extension of the range of ecological expansion of this species in Poland. The presence of I. parviflora may also result from the stands growing on former farmland in which this species had been present. No specimens of small balsam were found in the other experimental sites established in the fresh coniferous forest which confirms a thesis by Balcerkiewicz and Brzeg (1978) that mesotrophic rather than oligotrophic forest sites are at a greater risk of colonization by neophytic species.

The recorded results are almost completely consistent with the results of a survey conducted in the Wielkopolska region by Szwed and Perkiewicz (2010) which reported the dominance of species from the families of Asteraceae, Poaceae and Rosaceae. Along the road DW150 a lower share was recorded for species characteristic of the field communities from the class Stellarietea mediae which was outranked by the forest species from the class Querco-Fagetea. This fact is hardly surprising in view of the road vicinity. We also need to stress the presence of psammophilic grassland species from the class Koelerio glaucae-Corynephoretea canescentis with the dominant grey hairgrass. Sandy grassland together with heaths from the class Nardo-Callunetea constitute the substitute communities for the sub-Atlantic fresh coniferous forest within the so-called dynamic series of communities Leucobryo-Pinetum (Wojterska, 2003). The predominance of the local elements confirms further the thesis proposed by Balcerkiewicz and Brzeg (1978) on the susceptibility of communities to neophyte invasion depending on their trophic levels.

Literary sources mention several factors influencing diversification of the vegetation cover in the vicinity of transportation routes (see eg.: Bernhardt et al., 
2004; Corney et al., 2006; Delgado et al., 2007; Hawbaker et al., 2006; Suárez-Esteban et al., 2013). At the investigated road DW 150 a decisive role could have been played by light conditions, while in the immediate vicinity it may have also been accompanied by the diverse microrelief.

\section{CONCLUSIONS}

1. The recorded results are consistent with the literary data and confirm the formation of a characteristic boundary zone, ecotone, along a road with low traffic volume at the edges of pine stands. This zone differs significantly from the stand interior, e.g. in the more abundant occurrence and exceptional richness of species within the shrub and herbaceous layers and in the less developed lichen-moss layer.

2. The presented differences in the investigated characteristics in Poland are more evident in the case of poorer forest sites. Thus, they were particularly clearly manifested in the fresh coniferous forest site. Despite prospective differences in the distribution of the temperatures and natural lightning, no greater differences were observed in the formation of the ecotone zones depending on the stand exposure.

3. Despite the high proportion of plant species characteristic of synanthropic communities, no invasion of alien species (neophytes) was observed and the native species were dominant.

4. The boundary zone needs to be managed in an adequate and conscious manner, since it constitutes a buffer zone shielding stands located farther and it serves protective, biological, social and aesthetic roles. If feasible, the new road investment sites should be located at the edges of forest complexes taking advantage of the existing ecotone.

\section{REFERENCES}

Andres, B. (2005). Droga, jako czynnik odkształcający fitocenozy leśne [Road as factor determining forest phytocoenoses]. Sylwan, 5, 65-70 [in Polish].

Balcerkiewicz, S., Brzeg, A. (1978). Vegetation on the forest roads in pine forests. In: T. Wojterski (Ed.), Guide to the Polish International Excursion. International Society for Vegetation Science (pp. 115-117). Ser. Biology. Poznań: UAM.
Balcerkiewicz, S., Brzeg, A. (1993). Wrzosowiska przydrożne w kompleksie leśnym Borów Skwierzyńskich [Heath formations accompanying roads in the Bory Skwierzyńskie forest complex]. Bad. Fizjogr. Pol. Zach., Ser. B, 42, 105-127 [in Polish].

Balcerkiewicz, S., Kasprowicz, M. (1989). Wybrane aspekty synantropizacji szaty roślinnej ujawniające się na granicy kompleksów leśnych [Selected aspects of synanthropization of the vegetation cover at the border of forest complexes]. In: Wpływ gospodarki leśnej na środowisko. Seminarium naukowe. Sękocin, November 10-11 1988 (pp. 7-21). Warszawa: Wyd. SGGW-AR [in Polish].

Bernhardt, M., Fischer, A., Kirchner, M., Jakobi, G. (2004). Impact of motorways on adjacent coniferous forest communities. In: Otte A., D. Simmering, L. Eckstein, N. Hölzel, R. Waldhardt (Eds), Eco-complexity and dynamics of the cultural landscape. Proceedings of the 34th Annual Conference of the Ecological Society of Germany, Austria and Switzerland.

Bignal, K. L., Ashmore, M. R., Headley, A. D., Stewart, K., Weigert, K. (2007). Ecological impacts of air pollution from road transport on local vegetation. Appl. Geochem., 22, 1265-1271.

Brzeg, A. (1989). Przegląd systematyczny zbiorowisk okrajkowych dotąd stwierdzonych i mogących występować w Polsce [A systematic survey of 'saum'-communities found and possibly occurring in Poland]. Fragm. Flor. Geobot., 34 (3-4), 385-425 [in Polish].

Corney, P. M., Le Duc, M. G., Smart, S. M., Kirby, K. J., Bunce, R. G. H., Marrs, R. H. (2006). Relationships between the species composition of forest field-layer vegetation and environmental drivers, assessed using a national scale survey. J. Ecol., 94, 383-401.

Czępińska-Kamińska, D., Janowska, E., Zielony, R. (1997). Wpływ dróg szybkiego ruchu na środowisko glebowe i roślinność leśną [Influence of express roads on the soil and forest vegetation]. Mat. Konf. Nauk.-Tech. SITLiD “Autostrady a lasy” (pp. 1-6). Poznań [in Polish].

Czerniak, A., Poszyler-Adamska, A., Kayzer, D. (2007). Ocena propagacji hałasu komunikacyjnego na terenach zadrzewionych [Evaluation of traffic noise propagation in afforested areas]. Infrastruk. Ekol. Teren. Wiej., 1, 67-79 [in Polish].

Delgado, J. D., Arroyo, N. L, Arevalo, J. R., Fernández-Palacios, J. M. (2007). Edge effects of roads on temperature, light, canopy cover, and canopy height in laurel and pine forests (Tenerife, Canary Islands). Landscape Urban Plan., 81, 328-340. 
Mizera, P., Grajewski, S. M., Kasztelan, A. (2016). The floral characteristics of the road edge effect on the example of a road with low traffic volume in the "Puszcza Notecka" Forest. Acta Sci. Pol. Silv. Colendar. Ratio Ind. Lignar., 15(4), 297-312. DOI: 10.17306/J. AFW.2016.4.30

Eigenbrod, F., Hecnar, S. J., Fahrig, L. (2008). Accessible habitat: an improved measure of the effects of habitat loss and roads on wildlife populations. Landscape Ecol., $23,159-168$.

Falińska, K. (2004). Ekologia roślin [Plants ecology]. Warszawa: PWN [in Polish].

Faliński, J. B. (1961). Roślinność dróg leśnych w Białowieskim Parku Narodowym [The vegetation along forest roads in the Bialowieza National Park]. Acta Soc. Bot. Pol., 30, 163-185 [in Polish].

Faliński, J. B. (1995). Antropogeniczne przeobrażenia szaty roślinnej i ekosystemów [Anthropogenic transformation of plant cover and ecosystems]. In: Drągowski A. et al. (Eds), Antropogeniczne przemiany środowiska. Cz. 3 (pp. 69-96).Warszawa: WSiP [in Polish].

Forman, R. T. T., Alexander, L. E. (1998). Roads and their major ecological effects. Ann. Rev. Ecol. Syst., 29, 207-231.

Fukarek, F. (1967). Fitosocjologia [Phytosociology]. Warszawa: PWRiL [in Polish].

Garcia-Gonzaleza, C., Campoa, D., Polaa, I. G., GarciaVazqueza, E. (2012). Rural road networks as barriers to gene flow for amphibians: Species-dependent mitigation by traffic calming. Landscape Urban Plan., 104, 171-180.

Gibert, S. (1996). Sukcesja roślinna na szlakach zrywkowych w Nadleśnictwie Choszczno [Plant succession on skidding routes in Choszczno Forest Division]. Master Thesis, Faculty of Forestry, BG UP, Poznań [in Polish].

Hansen, M. J., Clevenger, A. P. (2005). The influence of disturbance and habitat on the presence of non-native plant species along transport corridors. Biol. Conserv., 125, 249-259.

Hawbaker, T. J., Radeloff, V. C., Clayton, M. K., Hammer, R. B., Gonzalez-Abraham, C. E. (2006). Road development, housing growth, and landscape fragmentation in northern Wisconsin: 1937-1999. Ecol. Appl., 16, 1222-1237.

Koivula, M. J., Vermeulen, H. J. W. (2005). Highways and forest fragmentation - effects on carabid beetles (Coleoptera, Carabidae). Landscape Ecol., 20, 911-926.

Kuitunen, M. T., Viljanen, J., Rossi, E., Stenroos, A. (2003). Impact of busy roads on breeding success in pied flycatchers Ficedula hypoleuca. Environ. Managem., 31, 79-85.

Kujawa-Pawlaczyk, J. (1991). Rozprzestrzenianie się i neofityzm Impatiens parviflora DC. w Puszczy Białowieskiej [Propagation and neophytism of Impatiens parviflora DC. in the Białowieża Forest]. Phytocoenosis, 3, 213-222.
Marcantonio, M., Rocchini, D., Geri, F., Bacaro, G., Amici, V. (2013). Biodiversity, roads, landscape fragmentation: Two Mediterranean cases. Appl. Geogr., 42, 63-72.

Mizera, P., Grajewski, S. (2016). Efekt brzegowy drogi a występowanie krzewinek $\mathrm{z}$ rodziny Ericaceae i zmienność pH gleb w Puszczy Noteckiej [The dependence of the road edge effect on the dwarf shrubs occurrence from the Ericaceae family and variability of pH soils in the Puszcza Notecka Forest]. Infrastr. Ekol. Teren. Wiej., 3/2, 867-881 [in Polish].

Paszek, I., Załuski, T. (1998). Flora naczyniowa dróg leśnych powierzchni badawczo-dydaktycznej w Czarnym Bryńsku [Vascular flora of forest roads of researcheducation area in Czarny Bryńsk]. In: J. Miądlikowska (Ed.), Materiały sympozjum i obrad sekcji 51 Zjazdu Polskiego Towarzystwa Botanicznego. UG, Gdańsk, September 15-19 1998 [in Polish].

Paszek, I., Załuski, T. (2000). Forest roads in the synanthropisation process. In: B. Jackowiak, W. Żukowski (Eds), Mechanisms of anthropogenic changes of the plant cover (pp. 249-257). Poznań: Bogucki Wyd. Naukowe.

Ratyńska, H., Szwed, W. (1996). Roślinność - strefy przejścia i granice [Vegetation - the transition zone and boundaries]. Wiad. Bot., 40, 21-28 [in Polish].

Rutkowski, L. (2011). Klucz do oznaczania roślin naczyniowych Polski niżowej [Determination key of vascular plants of Polish lowland]. Warszawa: PWN [in Polish].

Suárez-Esteban, A., Delibes, M, Fedriani, J. M. (2013). Unpaved road verges as hotspots of fleshy-fruited shrub recruitment and establishment. Biol. Conserv., 167, 50-56.

Szwed, W. Perkiewicz, F. (2010). Różnorodność florystyczna poboczy dróg wybranych gmin Wielkopolski [Floristic roadsides diversity in selected communes of Wielkopolska Region]. Biul. Parków Krajobr. Wielk., 16 (18), 68-89 [in Polish].

Szwed, W., Ratyńska, H., Danielewicz, W., Mizgajski, A. (1999). Przyrodnicze podstawy kształtowania marginesów ekologicznych w Wielkopolsce [Natural basis for shaping the ecological margins in Wielkopolska Region]. Pr. Kat. Bot. Leśn. AR Pozn., 1 [in Polish].

Valentukevičiene, M. (2016). Research of the influence of the pollutants from road surface runoff on small rivers water quality (case study from Lithuania, EU). Internatiomal Conference: Eco-TIRAS International Assoc. of River Keepers Leo Berg Educ. Found., at Moldova, March 10-12.2016. Vol. 1. 611-615.

Watkins, R. Z., Chen, J., Pickens, J., Brosofske, K. D. (2001). Effects of forest roads on understory plants in 
a managed hardwood landscape. Conserv. Biol., 17 (2), 411-419.

Wójciak, H. (2003). Porosty, mszaki, paprotniki. Flora Polski [Lichens, Bryophytes, Pteridophytes. Polish flora]. Warszawa: MULTICO [in Polish].

Wojterska, M. (2003). Struktura krajobrazów roślinnych Pojezierza Międzychodzko-Sierakowskiego [Vegetation landscapes structure of Międzychodzko-Sierakowskie Lakeland]. Poznań: Bogucki Wyd. Naukowe [in Polish].
Wysocki, C., Sikorski, P. (2009). Fitosocjologia stosowana w ochronie i kształtowaniu krajobrazu [Applied phytosociology in the protection and landscape development]. Warszawa: Wyd. SGGW [in Polish].

Ziarnek, K. (2000). The role of forest roads in the dynamics of the flora of Puszcza Bukowa. In: B. Jackowiak, W. Żukowski (Eds.), Mechanisms of anthropogenic changes of the plant cover (pp. 299-303). Poznań: Bogucki Wyd. Nauk.

\section{CHARAKTERYSTYKA FLORYSTYCZNA DROGOWEGO EFEKTU BRZEGOWEGO NA PRZYKŁADZIE DROGI O BARDZO MAŁYM NATĘŻENIU RUCHU W PUSZCZY NOTECKIEJ}

\section{ABSTRAKT}

Zbadano oddziaływanie drogi na skład gatunkowy szaty roślinnej sąsiadujących z nią drzewostanów Puszczy Noteckiej. Obserwacje przeprowadzono w drzewostanach sosnowych w siedlisku boru świeżego i boru mieszanego świeżego. Założono 12 prostopadłych do osi drogi prostokątnych powierzchni doświadczalnych - transektów pasowych. Na każdym z nich wyznaczono 3-4 działki badawcze zlokalizowane w różnej odległości od krawędzi korony drogi. W toku inwentaryzacji flory rozpoznano łącznie 108 taksonów, z czego 70\% występowało wyłącznie w bezpośrednim sąsiedztwie drogi, 25\% spotykano zarówno przy drodze, jak i wewnątrz drzewostanu, a 5\% wyłącznie wewnątrz drzewostanu. W najbliższym sąsiedztwie drogi zauważono bardziej obfite występowanie podszytu oraz roślinności zielnej, rzadsze mchów i porostów. Mimo znacznego udziału roślin synantropijnych, nie stwierdzono inwazji gatunków obcych. Ze względu na małe natężenie ruchu i brak zabiegów utrzymaniowych nawierzchni drogi przyczyn zmian składu gatunkowego upatruje się głównie w modyfikacji warunków świetlnych i wilgotnościowych.

Słowa kluczowe: drogi leśne, sosna zwyczajna, bory sosnowe, szata roślinna 